\title{
Antidesma montanum: biochemistry and bioactive compounds
}

\begin{abstract}
Antidesma montanum is commonly known as a mountain currant tree. Its fruits and leaves are utilized locally for food and traditional medicine, meanwhile the stem is used as source of wood. The fruit contains bioactive constituents of steroid glycosides, saponins, flavonoids, polyphenols and tannins that exhibited higher antioxidant activity than at certain concentrations of standard antioxidants (i.e. vitamin E, ascorbic acid, and trolox). The leave extracts known to inhibit the activities of $\alpha$-amylase and $\alpha$-glucosidase in diabetic therapy. Furthermore, the leave extracts also possessed scavenging activity against both hydroxyl and superoxide anion radicals, and inhibited the nitric oxide production. Meanwhile, another studies reported on the anti-inflammatory effect of Antidesma montanum, being used to treat eye diseases, and relieving chest pain. The leaves are used externally against headache and thrush in children, for diuretic and removing kidney stone, anti-dermatitis and skin disease curing effect. A tea from the leaves is used as a tonic for mothers after giving birth and applied topically to ulcers and lumbar pains. The roots are used to treat measles, chickenpox, malaria, and stomach ache. Nevertheless, extensive research is needed to confirm the reputed beneficial effects of Antidesma montanum in traditional medicine.
\end{abstract}

Keyword: Antidesma montanum; Currant tree; Traditional medicine; Antioxidant; Antiinflammatory; Diabetic 